\title{
La variante SARS-CoV-2 ómicron.
}

\author{
SARS-CoV-2 omicron variant.
}

José Eduardo Orellana-Centeno, * Roxana Nayeli Guerrero Sotelo ${ }^{\ddagger}$

\section{RESUMEN}

Introducción: A finales de noviembre de 2021, la Organización Mundial de la Salud (OMS) designó a la cepa conocida como B.1.1.529 como una variante de preocupación y le otorgó la designación ómicron, con lo cual se convierte en la cuarta variante de preocupación enumerada, junto con las variantes alfa, beta, gamma y delta. El propósito de este estudio es presentar información de la aparición, características y algunos datos de la variante ómicron. Conclusiones: Las deficientes infraestructuras en los sistemas de salud que hay en nuestro país, la baja tasa de vacunación y la presencia de comorbilidades e inmunodeficiencia en la población inducen a que ésta sea más susceptible a la infección de estas nuevas variantes. Se requiere un esfuerzo global del gobierno, las industrias farmacéuticas/biotecnológicas y las instituciones académicas y sanitarias para contener eficazmente esta pandemia.

Palabras clave: COVID-19, ómicron, México.

\section{ABSTRACT}

Introduction: In late November 2021, the World Health Organization (WHO) designated the strain known as B.1.1.529 as a variant of concern, and granted it the omicron designation, making it the fourth variant of concern listed along with alpha, beta, gamma, and delta variants. The purpose of the study is to present information on the occurrence, characteristics and some data on the omicron variant. Conclusions: The deficient health infrastructures present in our country, the low vaccination rate and the presence of population with comorbidities and immunodeficiency or both, are more susceptible to infection to these new variants. A global effort by the government, pharmaceutical/biotechnology industries, and academic and health institutions is required to effectively contain this pandemic.

Keywords: COVID-19, omicron, Mexico.

\section{INTRODUCCIÓN}

$\mathrm{E}$ I día 26 de noviembre del año 2021, la Organización Mundial de la Salud (OMS), a través del consejo de científicos que forman parte del Grupo de Asesoramiento Técnico de la OMS, designó a la cepa conocida como B.1.1.529 como una variante de preocupación y le dio la denominación ómicron, con ello se convertía en la cuarta variante de preocupación en la lista junto a las variantes alfa, beta, gamma y delta.

La variante ómicron apareció por primera vez en Botsuana y se ha extendido en varios países africanos con una mayor extensión en Sudáfrica. Esta variante alberga un gran número de mutaciones que se manifiestan inclusive en otras variantes.

El propósito del estudio es presentar información de la aparición, características y algunos datos de la variante ómicron.

\section{CAMBIOS ESTRUCTURALES}

La variante ómicron se destaca porque presenta más de 30 cambios identificados en la proteína spike, que es la encargada de reconocer las células del huésped y es el principal objetivo de las respuestas inmunitarias del organismo. Este tipo de cambios pretenden una mayor

\footnotetext{
* Licenciatura en Odontología. Doctorante en Educación, Maestro en Salud Pública. Profesor Investigador de tiempo completo.

‡ Licenciatura en Enfermería, Doctora en Ciencias Sociales. Profesora Investigadora de tiempo completo.

Universidad de la Sierra Sur, Instituto de Investigación sobre la Salud Pública. México.

Recibido: 13 de diciembre de 2021. Aceptado: 14 de diciembre de 2021.

Citar como: Orellana-Centeno JE, Guerrero SRN. La variante SARS-CoV-2 ómicron. Rev ADM. 2022; 79 (1): 28-31. https://dx.doi.org/10.35366/103815
}

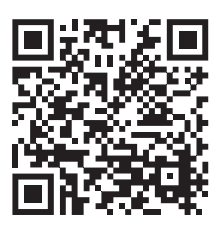


infectividad y capacidad de evadir los anticuerpos que bloquean la infección. Del mismo modo, no presentan dificultad para respirar, lo que hace pensar a los expertos en una variante con una sintomatología menor.

En Sudáfrica los científicos iniciaron sus primeros estudios de identificación de esta nueva variante teniendo la experiencia de su rápida movilización en la variante beta, lo que han observado hasta el momento es mayor infección en el grupo etario entre 20 y 40 años de edad. ${ }^{1}$

La variante alberga un número de mutaciones en regiones de la proteína spike que los anticuerpos reconocen, y en algunos modelos informáticos predicen que podría eludir la inmunidad conferida por otro componente del sistema inmunitario llamado células T. Farmacéuticas e investigadores independientes continúan los estudios para obtener los primeros resultados que permitan conocer un poco más de la infectividad y otras características virológicas y patogénicas de esta variante. ${ }^{2}$

\section{SÍNTOMAS}

Esta nueva variante, a diferencia de las anteriores, no presenta falta del sentido del olfato ni ausencia del gusto como anteriormente en el COVID-19. ${ }^{3}$ Los síntomas más recurrentes son: cansancio y dolor muscular, fiebre, tos seca, dolor de garganta, dolor de cabeza y taquicardia.

\section{TRANSMISIBILIDAD}

Aún no se tiene evidencia suficiente para afirmar que es más transmisible, aunque lo observado en Sudáfrica nos hace pensar que así es. Pero se debe continuar con los seguimientos de vigilancia epidemiológica para llegar a una conclusión.

Es posible que las múltiples mutaciones del virus lo hagan menos virulento, la probabilidad es muy pequeña. Por el contrario, el carácter altamente infeccioso de esta variante sugiere que, una vez que un individuo está infectado, produzca una propagación más amplia y rápida, por lo tanto patologías y manifestaciones de la enfermedad aún tienen un pronóstico reservado. ${ }^{4}$

Las mutaciones en Nsp12-RdRp y Nsp14-Exo virales confieren una mayor tasa de mutación en la variante ómicron. Mediante la mutación y la adaptación, las variantes virales pueden evadir eficazmente la inmunidad del huésped, lo que da lugar a una infección prolongada y a la propagación del virus a otras personas.

Todavía se estudia el cuadro clínico de la infección, no se puede asegurar que es más grave con respecto a otras variantes, lo cierto es que han aumentado las tasas de hospitalización en países como Sudáfrica, hay que tomar con cautela este dato, ya que podría deberse a que las personas han contraído la infección, pero no necesariamente que se hayan infectado de dicha variante. ${ }^{5}$

\section{CASO EN MÉXICO}

El primer caso registrado en México fue del sexo masculino con edad de 51 años, de origen sudafricano, con esquema de vacunación completo con la vacuna PfizerBioNTech que ingresó a México el día 21 de noviembre de 2021. El día 27 de noviembre de 2021 presentó un cuadro de COVID-19 leve con los siguientes signos y síntomas: tos, malestar generalizado y fiebre. Es hospitalizado en la Ciudad de México en un hospital privado, donde se le realizaron las pruebas de antígeno y PCR, las cuales dieron positivas. El día 30 de noviembre del presente año fueron enviados los resultados al INDRE (Instituto de Diagnóstico y Referencia Epidemiológicos), donde se confirma que es la variante ómicron.

\section{DERECHOS HUMANOS}

Las primeras variantes de la COVID-19 y sus olas de contagio visibilizaron pública e internacionalmente la fragilidad y la precariedad con la que opera el Sistema Nacional de Salud (SNS) en México, no sólo por la escasez de recursos materiales, financieros y humanos, sino también por los fenómenos asociados a una mala o pésima administración de la pandemia, y una incapacidad grave de reconocer y/o corregir errores por parte de la administración pública central. ${ }^{6}$

Es en este terreno que las muertes asociadas al día 9 de diciembre de 2021, según las cifras oficiales, son de $442,615,{ }^{7}$ a lo que se suma un aumento de $43.9 \%$ de pobres en 2020 respecto de 2018, quedando en 55.7 millones de personas que viven en pobreza y $8.5 \%$ de aumento en la pobreza extrema equivalente a $10.8 \mathrm{mi}-$ llones de personas. Esto relacionado con los derechos humanos implicó un aumento en las personas que no tienen al acceso al sistema de salud quedando en 2020 con 35.7 millones de personas. ${ }^{8}$ En Oaxaca la carencia de los servicios de salud en 2020 asciende a $36.9 \%$ de su población, equivalente a 1,538,900 personas, lo que coloca a este estado en el tercer lugar de la República. ${ }^{9}$ También ocupa el mismo lugar en pobreza extrema con $28.7 \%$ de su población equivalente a 1,196,900 personas.

Es en estas circunstancias socioeconómicas que llega ómicron a México, lo que significa directamente un 
reto ligado al cumplimiento del derecho humano a la salud, e indirectamente a los derechos humanos que le son interdependientes, que grosso modo se identifican con los derechos económicos, sociales y culturales, pero también con los civiles y políticos. ${ }^{10}$ En este sentido, el Secretario General de la ONU ha expresado el inicio de la «pandemia de abusos contra los derechos humanos», pues ha profundizado la pobreza, la vulnerabilidad y la desigualdad preexistentes, ${ }^{11,12}$ y que en los países en desarrollo se recrudece por los fenómenos de desigualdad social, étnica (interculturalidad) y de género.

Los debates de derechos humanos en torno a COVID-19 tienen diversas aristas, por la importancia de los problemas tratados podemos enumerar los siguientes: la exención o liberación de la patente de vacunas y medicamentos; la garantía a un acceso universal a medicamentos, vacunas y tecnologías sanitarias como parte del derecho humano a la salud; los derechos del personal sanitario; la igualdad jurídica de los profesionales de la salud; los derechos del personal sanitario en servicio social y residencias; la eficacia de los estados de derecho, entre otros.

El descubrimiento de la variante ómicron se acompañó de una propuesta jurídica innovadora y es la exención del derecho de propiedad intelectual de las patentes respecto de las diversas fórmulas de las vacunas. A pesar de que esta medida permitiría realizar una distribución equitativa del recurso y lograr una inmunización mundial más extensa, la propuesta ha sido rechazada, lo que impide la universalización del know-how. ${ }^{13}$

\section{BIOÉTICA}

La aparición de la variante ómicron visibilizó que la bioética imperante en el mundo, ya sea de corte principialista (autonomía, beneficencia, no maleficencia y justicia) o personalista (principios de defensa de la vida física, terapéutica, libertad, responsabilidad, sociabilidad y solidaridad) resulta cuestionable desde el punto de vista ético, en razón de que el modelo de salud que orienta perpetúa tanto el colonialismo de ciertas partes geográficas del mundo, como es el caso de África, Latinoamérica, el Caribe y Asia, así como la explotación y cosificación del ser humano pasando de ser un fin en sí mismo a un medio para obtener otro fin.

Esta situación ha sido denunciada internacionalmente desde muchas latitudes y desde distintas perspectivas, entre ellas destacan las críticas tanto de la OMS como las Human Right Watch, ${ }^{13}$ ambos coinciden en los siguientes argumentos: la necesidad de vacunar a la mayoría de la población mundial (Europa con 50\% de vacunación y
África 7\%); el incremento de pobreza y pobreza extrema asociada a la pandemia en la mayoría de los países en desarrollo; la exención de los derechos de patente de las farmacéuticas transnacionales sobre vacunas y fármacos; y la consideración de bienes públicos para la salud.

Además del aspecto general de la bioética en relación con la pandemia de COVID-19, es necesario pensar cuál ha sido su impacto en la odontología, grosso modo podemos identificar que el gremio fue vacunado posteriormente a la fecha asignada a otros profesionales de la salud como médicos y enfermeras, ${ }^{14}$ situación replicada en otros países como España. Asimismo, por la integración pluricultural del Estado mexicano es factible que los problemas identificados con el paternalismo biomédico se acentuarán en las relaciones odontólogo-paciente en la pandemia COVID-19 como la asimetría de saberes y la subordinación del paciente. ${ }^{15}$

Por último, es necesario reconocer que ha habido un gran esfuerzo del gremio estomatológico para desarrollar directrices, manuales y normas que regulen el comportamiento del odontólogo y del paciente en circunstancias de alto riesgo epidemiológico. De esta forma, a los Principios de ética y al Código de Conducta Profesional de la Asociación Dental Americana (ADA) se sumaron para la atención de la salud bucodental las recomendaciones del Subcomité de Ética del Consejo de Ética, Estatutos y Asuntos Judiciales de la ADA, y entre sus principales directrices destacan: sólo tratar emergencias dentales, uso adecuado del equipo de protección personal, reducir el uso de aerosoles, reducción del contacto cercano entre paciente y realizar pruebas de detección de COVID-19 constantemente. ${ }^{16,17}$

\section{CONCLUSIÓN}

La Organización Mundial de la Salud (OMS) hizo recomendaciones a los países miembros: mantener el distanciamiento social, salir a la calle sólo por cuestiones laborales o compra de alimentos, medicamentos y enseres necesarios para la vida cotidiana, evitar salir por cuestiones distintas a ello y, sobre todo, a lugares donde pueda acumularse un gran número de personas (cine, restaurantes, eventos masivos, etc.); si es necesario abandonar la vivienda para actividades de tipo esencial, recordar que hay que dejar una distancia de aproximadamente de 1.5 a 2 metros entre cada persona. Entre otras recomendaciones se encuentran el lavado de manos con agua y jabón o gel antibacterial, evitar tocarse la cara con la mano y limpiar continuamente las superficies. ${ }^{18}$

Se deben esperar los estudios y las pruebas que están realizando las farmacéuticas creadoras de las vacunas para 
tener mayor información sobre la trasmisibilidad, cuadros clínicos y conocimiento más amplio de las variantes sin crear especulaciones y siendo muy objetivos para beneficio y conocimiento de quienes toman decisiones y de la población.

México y Colombia son los únicos dos países que no tienen restricciones a los visitantes que llegan a los aeropuertos, en los demás países existen restricciones parciales con algunos o varios de los requisitos a continuación detallados: prueba negativa de PCR 72 a 48 horas previo al vuelo, certificado o comprobante de esquema completo de vacunación, prueba al arribo de PCR o antígeno, prohibición de viajeros provenientes de Sudáfrica, cuarentena de 10 a 14 días de pasajeros provenientes de Sudáfrica.

Las deficientes infraestructuras en salud que hay en nuestro país, la baja tasa de vacunación y la presencia de comorbilidades e inmunodeficiencia, o ambas, en la población inducen a que ésta sea más vulnerable a la infección de estas nuevas variantes. Se requiere un esfuerzo global del gobierno, las industrias farmacéuticas/ biotecnológicas y las instituciones académicas y sanitarias para contener eficazmente esta pandemia.

\section{REFERENCIAS}

1. Gao SJ, Guo H, Luo G. Omicron variant (B.1.1.529) of SARS-CoV-2, a global urgent public health alert! J Med Virol. 2021. doi: 10.1002/ jmv.27491.

2. Callaway E, Ledford H. How bad is omicron? What scientists know so far. Nature. 2021; 600 (7888): 197-199. doi: 10.1038/d41586021-03614-z.

3. Callaway E. Heavily mutated Omicron variant puts scientists on alert. Nature. 2021; 600 (7887): 21. doi: 10.1038/d41586-02103552-w.

4. Dyer O. Covid-19: South Africa's surge in cases deepens alarm over omicron variant. BMJ. 2021; 375: n3013. doi: 10.1136/bmj. n3013.

5. Daria S, Bhuiyan MA, Islam MR. Detection of highly muted coronavirus variant Omicron (B.1.1.529) is triggering the alarm for South Asian countries: Associated risk factors and preventive actions. J Med Virol. 2021. doi: 10.1002/jmv.27503.

6. Sánchez-Talanquer M, González-Pier E, Sepúlveda J, Abascal-Miguel L, Fieldhouse J, Del Río C et al. La respuesta de México al COVID-19: estudio de caso. Institute for Global Health Sciences. Disponible en: https://globalhealthsciences.ucsf.edu/sites/globalhealthsciences. ucsf.edu/files/la_respuesta_de_mexico_al_covid_esp.pdf
7. Secretaria de Salud (SSA). Exceso de mortalidad en México. Disponible en: https://coronavirus.gob.mx/exceso-de-mortalidaden-mexico/

8. Consejo Nacional de Evaluación de la Política de Desarrollo Social (CONEVAL). Medición de la pobreza 2016-2020, México. Disponible en: https://www.coneval.org.mx/Medicion/Paginas/ Pobrezalnicio.aspx

9. Consejo Nacional de Evaluación de la Política de Desarrollo Social (CONEVAL). Medición de la pobreza 2020, México. Disponible en: https://www.coneval.org.mx/Medicion/Paginas/Resultados_ Pobreza Interactivo.aspx

10. Guerrero Sotelo RN, Orellana Centeno JE. El derecho humano a la salud: un estudio desde el derecho crítico. Revista Direito e Práxis. 2021. Disponible en: https://www.e-publicacoes.uerj.br/index.php/ revistaceaju/article/view/56214

11. Organización de Naciones Unidas (ONU). The world faces a pandemic of human rights abuses in the wake of Covid-19. Available in: https://www.un.org/sexualviolenceinconflict/press/ the-world-faces-a-pandemic-of-human-rights-abuses-in-the-wakeof-covid-19/

12. Donald A, Leach P. Human rights and COVID-19: forging recovery after a pandemic of abuses? VerfBlog; 2021. Available in: https:// verfassungsblog.de/human-rights-and-covid-19-forging-recoveryafter-a-pandemic-of-abuses/

13. Hassan T. Los derechos humanos y los estados ante la excepción de patentes COVID. El País. Diciembre 3, 2021. Disponible en: https://www.hrw.org/es/news/2021/12/03/los-derechos-humanosy-los-estados-ante-la-exencion-de-patentes-covid

14. Senado de la República de México. Proponen vacunar contra COVID-19 a las y los odontólogos. 20 de febrero de 2021. Disponible en: http:/comunicacion.senado.gob.mx/index.php/ informacion/boletines/50327-proponen-vacunar-contra-covid-19a-las-y-los-odontologos.html

15. Orellana Centeno JE, Guerrero Sotelo RN. La bioética desde la perspectiva odontológica. Rev ADM. 2019; 76 (5): 282-286.

16. Ethics Subcommittee of the Council on Ethics, Bylaws and judicial affairs. Ethical practice during the COVID-19 pandemic. J Am Dent Assoc. 2020; 151 (5): 377-378. doi: 10.1016/j.adaj.2020.03.038.

17. Wilson $\mathrm{R}$, Jonke $\mathrm{G}$. The ethics of dentists receiving the COVID-19 vaccine: Following the American Dental Association Principles of Ethics and Code of Professional Conduct. J Am Dent Assoc. 2021; 152 (5): 408-409. doi: 10.1016/j.adaj.2021.02.011.

18. Orellana-Centeno JE, Guerrero-Sotelo RN. Impacto socioeconómico de la COVID-19 en el escenario mexicano. Acta Odont Col. 2021; 11 (2): 127-135. doi: 10.15446/aoc.v11n2.97196.

Financiamiento: Ningún financiamiento fue recibido para el desarrollo de la presente investigación.

Conflicto de intereses: Los autores manifiestan que no existe ningún tipo de conflicto de intereses.

Correspondencia:

José Eduardo Orellana-Centeno

E-mail: jeorellano@unsis.edu.mx 